\title{
State Estimation for Legged Robots - Consistent Fusion of Leg Kinematics and IMU
}

\author{
Michael Bloesch, Marco Hutter, Mark A. Hoepflinger, Stefan Leutenegger, Christian Gehring, \\ C. David Remy and Roland Siegwart \\ Autonomous Systems Lab, ETH Zürich, Switzerland, bloeschm@ethz.ch
}

\begin{abstract}
This paper introduces a state estimation framework for legged robots that allows estimating the full pose of the robot without making any assumptions about the geometrical structure of its environment. This is achieved by means of an Observability Constrained Extended Kalman Filter that fuses kinematic encoder data with on-board IMU measurements. By including the absolute position of all footholds into the filter state, simple model equations can be formulated which accurately capture the uncertainties associated with the intermittent ground contacts. The resulting filter simultaneously estimates the position of all footholds and the pose of the main body. In the algorithmic formulation, special attention is paid to the consistency of the linearized filter: it maintains the same observability properties as the nonlinear system, which is a prerequisite for accurate state estimation. The presented approach is implemented in simulation and validated experimentally on an actual quadrupedal robot.
\end{abstract}

\section{INTRODUCTION}

Particularly in rough and highly unstructured environments in which we ultimately want to employ autonomous legged robots, postural controllers require fast and precise knowledge of the state of the robots they are regulating. Especially for dynamic locomotion, the underlying state estimation can quickly become a bottleneck in terms of achievable bandwidth, robustness, and locomotion speed. To achieve the required performance, a state estimator for legged robots should explicitly take into account that such systems are interacting with their environment via multiple intermittent ground contacts. Ignoring or neglecting the ground interaction will lead to computationally and sensory more "expensive" approaches, ranging from vision-based [2, 16, 17] to GPS-supported [4, 6] methods. In contrast to such approaches, we will show in the following that in cases where on-board sensors fully measure the internal kinematics of the robot as well as its inertial acceleration and rotational rate, precise information on the robot's pose can be made readily available.

One of the earliest approach exploiting information given by the leg kinematics was implemented by Lin et al. [13] in 2005 on a hexapod robot. Assuming that the robot is in contact with three of its six feet at all times and assuming completely flat terrain, they implemented a leg-based odometer. Their method requires the robots to follow a tripod gait and is affected by drift. In [14], the same group fused the leg-based odometer with data from an Inertial Measurement Unit (IMU) and thus is able to handle tripod running. Using the assumption of knowing the precise relief of the terrain, Chitta et al. [3] implemented a pose estimator based on a particle filter. It fuses

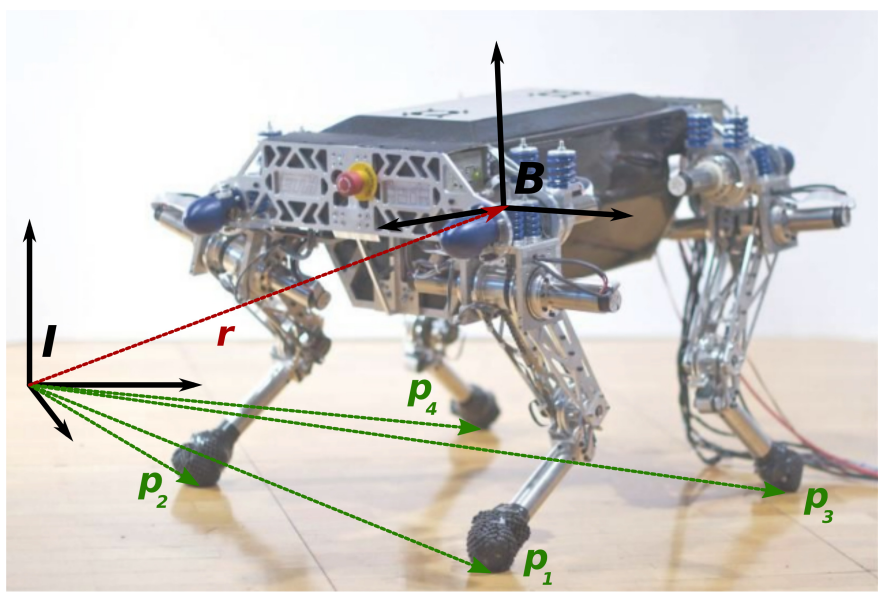

Fig. 1. Experimental quadruped platform StarlETH [11]. The inertial and the body fixed coordinate frames $\boldsymbol{I}$ and $\boldsymbol{B}$ are depicted, as well as the absolute position vectors of the robot $\boldsymbol{r}$ and of the footholds $\boldsymbol{p}_{1}, \ldots, \boldsymbol{p}_{N}$. The presented EKF includes all foothold positions into the estimation process.

leg kinematics and IMU in order to globally localize a robot.

Just very recently, three novel pose estimators have been presented that are all based on leg kinematics. Reinstein and Hoffmann [15] presented a data-driven approach using joint encoders, pressure sensors, and an on-board IMU. They searched for significant sensory based indicators in order to determine the stride length when given a specific gait pattern. With this assumption, they successfully limited the position drift of the IMU and by appropriate training of the filter could additionally handle slippage. Chilian et al. [2] implemented a leg odometer based on point cloud matching for a hexapod robot, requiring a minimum of three feet in contact. It is based on a multisensor fusion algorithm that includes inertial measurements and visual odometry. Assuming planar springmass running, Gur and Saranli [7] proposed a generic, modelbased state estimation technique.

In the presented approach we implement an Extended Kalman Filter (EKF) and choose an appropriate state vector in order to break down the estimation problem to the proper formulation of a few simple measurement equations. Without any assumption about the shape of the terrain, we are able to estimate the full state of the robot's main body, and we can provide an estimate of the ground geometry. By performing an observability analysis, we show that apart from the absolute position and yaw angle of the robot all other states can be precisely observed as long as at least one foot is in contact 
with the ground. This means that, after accumulating some drift during a flight phase, the pitch and roll angles become again fully observable when the robot regains ground contact and the corresponding estimation errors will decrease.

Only proprioceptive sensors are required and no assumptions are made concerning the type of gait or the number of robot legs. Little foot slippage and uncertainties on the leg kinematics can be handled as well. Due to current limitations of the control approach, dynamic gaits are currently evaluated in simulation only. Still, results obtained from static walking sequences on an actual quadrupedal platform (see Fig. 1) are presented and compared with ground truth measurements from an external motion tracker.

The structure of the paper is as follows. In section II a short overview of the sensory devices is provided. Subsequently, section III presents the design of an Extended Kalman Filter. Section IV argues on the observability of the filter states and introduces observability constraints. Simulation and experimental validation are discussed in section $\mathrm{V}$

\section{Sensor Devices and Measurement Models}

This section discusses the required sensors and the corresponding stochastic measurement models for a $N$ legged robot. The particular model choices represent a trade-off between simplicity and accuracy. Throughout the paper, external disturbances and noise will be modeled as continuous white Gaussian noise or as discrete Gaussian noise processes. This is a coarse simplification, but can be handled by increasing the corresponding covariance matrix.

\section{A. Forward Kinematics and Encoders}

Incremental encoders provide access to the angular position of all joints. The corresponding encoder measurement vector $\tilde{\boldsymbol{\alpha}}$ of the joint angles vector $\boldsymbol{\alpha}$ is assumed to be affected by discrete Gaussian noise $\boldsymbol{n}_{\alpha}$ with covariance matrix $\boldsymbol{R}_{\alpha}$ :

$$
\tilde{\boldsymbol{\alpha}}=\boldsymbol{\alpha}+\boldsymbol{n}_{\alpha} .
$$

Based on the known leg kinematics, the location of each foot can be computed with respect to the main body. However, due to erroneous calibration and possible errors in the kinematical $\operatorname{model} \operatorname{lkin}_{i}(\cdot)$ of leg $i$, additive discrete Gaussian noise terms $\boldsymbol{n}_{s, i}$ are included in the model:

$$
\boldsymbol{s}_{i}=\operatorname{lkin}_{i}(\boldsymbol{\alpha})+\boldsymbol{n}_{s, i},
$$

where $s_{i}$ represents the vector from the center of the main body to the contact point of leg $i$ and where $\boldsymbol{R}_{s}$ is the covariance matrix of $\boldsymbol{n}_{s, i}$. Both, $\boldsymbol{s}_{i}$ and $\boldsymbol{n}_{s, i}$, are expressed in the body fixed frame $\boldsymbol{B}$.

\section{B. Inertial Sensors}

The IMU measures the proper acceleration $f$ and the angular rate $\boldsymbol{\omega}$ of the robot's main body. The proper acceleration is related to the absolute acceleration $\boldsymbol{a}$ by

$$
\boldsymbol{f}=\boldsymbol{C}(\boldsymbol{a}-\boldsymbol{g}),
$$

where $C$ is the matrix rotating coordinates of a vector expressed in the inertial coordinate frame $\boldsymbol{I}$ into the body coordinate frame $\boldsymbol{B}$. The IMU quantities $\boldsymbol{f}$ and $\boldsymbol{\omega}$ are assumed to be directly measured in the body coordinate frame $\boldsymbol{B}$. In order to describe the underlying stochastic process, the following continuous stochastic models are introduced:

$$
\begin{aligned}
\tilde{\boldsymbol{f}} & =\boldsymbol{f}+\boldsymbol{b}_{f}+\boldsymbol{w}_{f}, \\
\dot{\boldsymbol{b}}_{f} & =\boldsymbol{w}_{b f}, \\
\tilde{\boldsymbol{\omega}} & =\boldsymbol{\omega}+\boldsymbol{b}_{\omega}+\boldsymbol{w}_{\omega}, \\
\dot{\boldsymbol{b}}_{\omega} & =\boldsymbol{w}_{b \omega} .
\end{aligned}
$$

The measured quantities $\tilde{f}$ and $\tilde{\omega}$ are affected by additive white Gaussian noise processes $\boldsymbol{w}_{f}$ and $\boldsymbol{w}_{\omega}$ and by bias terms $\boldsymbol{b}_{f}$ and $\boldsymbol{b}_{\omega}$. The bias terms are modeled as Brownian motions and their derivatives can be represented by white Gaussian noise processes $\boldsymbol{w}_{b f}$ and $\boldsymbol{w}_{b \omega}$. The noise terms are specified by the corresponding covariance parameters $\boldsymbol{Q}_{f}, \boldsymbol{Q}_{b f}, \boldsymbol{Q}_{\omega}$, and $\boldsymbol{Q}_{b \omega}$. Following the paper of El-Sheimy et al. [5], they can be evaluated by examining the measured Allan variances. For the sake of simplicity each covariance parameter is assumed to be a diagonal matrix with identical diagonal entries.

\section{State Estimation}

As stated in the previous section, two different sources of data are available. Each of them provides information that can potentially contribute to the state estimate of the robot. In order to exploit this information an Extended Kalman Filter is designed. This section starts by defining the state vector of the filter and subsequently continues by formulating the filter models and equations.

\section{A. Filter State Definition}

The state vector of the filter has to be chosen such that the corresponding prediction and measurement equations can be stated in a clean and consistent manner. In this approach the state vector of the quadruped robot is composed of the position of the center of the main body $\boldsymbol{r}$, of the corresponding velocity $\boldsymbol{v}$ and of the quaternion $\boldsymbol{q}$ representing the rotation from the inertial coordinate frame $\boldsymbol{I}$ to the body coordinate frame $\boldsymbol{B}$. In order to consider the kinematics of the legs, the absolute positions of the $N$ foot contact points $\boldsymbol{p}_{i}$ are included into the state vector. Together with the accelerometer bias $\boldsymbol{b}_{f}$ and the gyroscope bias $\boldsymbol{b}_{\boldsymbol{\omega}}$ this yields the following state vector:

$$
\boldsymbol{x}:=\left(\begin{array}{llllllll}
\boldsymbol{r} & \boldsymbol{v} & \boldsymbol{q} & \boldsymbol{p}_{1} & \cdots & \boldsymbol{p}_{N} & \boldsymbol{b}_{f} & \boldsymbol{b}_{\omega}
\end{array}\right) .
$$

$\boldsymbol{r}, \boldsymbol{v}$ and all contact positions $\boldsymbol{p}_{i}$ are expressed in the inertial coordinate frame $\boldsymbol{I}$, whereas $\boldsymbol{b}_{f}$ and $\boldsymbol{b}_{\omega}$ are expressed in the body coordinate frame $\boldsymbol{B}$. Given a quaternion $\boldsymbol{q}$ the corresponding rotation matrix $C$ can be easily determined.

The presented Extended Kalman Filter represents the uncertainties of the estimated state vector via the covariance matrix $\boldsymbol{P}$ of the corresponding state error vector $\boldsymbol{\delta} \boldsymbol{x}$

$$
\begin{aligned}
& \boldsymbol{P}:=\operatorname{Cov}(\boldsymbol{\delta} \boldsymbol{x}), \\
& \delta x:=\left(\begin{array}{llllllll}
\delta r & \delta v & \delta \phi & \delta p_{1} & \cdots & \delta p_{N} & \delta b_{f} & \delta b_{\omega}
\end{array}\right) .
\end{aligned}
$$


For the orientation state $\boldsymbol{q}$, special care has to be taken. It possesses 3 degrees of freedom and its covariance term should therefore also be represented by a 3 dimensional covariance matrix. Therefore the error of the pose is represented as a 3dimensional rotation vector $\boldsymbol{\delta} \phi$. That is, if $\hat{\boldsymbol{q}}$ represents the estimate of the orientation quaternion, the error quaternion $\boldsymbol{\delta} \boldsymbol{q}$ is defined by the relation

$$
\boldsymbol{q}=\boldsymbol{\delta} \boldsymbol{q} \otimes \hat{\boldsymbol{q}},
$$

where $\otimes$ is the quaternion multiplication operator and where the quaternion error is related to the error rotation vector by means of the map $\zeta(\cdot)$ :

$$
\begin{aligned}
\boldsymbol{\delta} \boldsymbol{q} & =\boldsymbol{\zeta}(\boldsymbol{\delta} \boldsymbol{\phi}) \\
\boldsymbol{\zeta}: \boldsymbol{v} \mapsto \boldsymbol{\zeta}(\boldsymbol{v}) & =\left[\begin{array}{c}
\sin \left(\frac{1}{2}\|\boldsymbol{v}\|\right) \frac{\boldsymbol{v}}{\|\boldsymbol{v}\|} \\
\cos \left(\frac{1}{2}\|\boldsymbol{v}\|\right)
\end{array}\right] .
\end{aligned}
$$

The inclusion of the foot contact positions into the filter state is the key point in the filter design, enabling a simple and consistent representation of the model equations. The leg kinematics measurements represent relative pose measurements between main body and foot contact, based on which the EKF is able to simultaneously correct the location of the foot contacts as well as the pose of the main body. In fact, the presented approach can be interpreted as a simultaneous localization and mapping (SLAM) algorithm, where the position of the actual foot contacts build up the map the robot is localized in.

\section{B. Prediction model}

The prediction equations are responsible for propagating the state from one timestep to the next. The IMU measurements $\tilde{f}$ and $\tilde{\boldsymbol{\omega}}$ are directly included here. Using $(3)-(7)$, a set of continuous time differential equations can be formulated:

$$
\begin{aligned}
\dot{\boldsymbol{r}} & =\boldsymbol{v} \\
\dot{\boldsymbol{v}} & =\boldsymbol{a}=\boldsymbol{C}^{T}\left(\tilde{\boldsymbol{f}}-\boldsymbol{b}_{f}-\boldsymbol{w}_{f}\right)+\boldsymbol{g}, \\
\dot{\boldsymbol{q}} & =\frac{1}{2} \boldsymbol{\Omega}(\boldsymbol{\omega}) \boldsymbol{q}=\frac{1}{2} \boldsymbol{\Omega}\left(\tilde{\boldsymbol{\omega}}-\boldsymbol{b}_{\omega}-\boldsymbol{w}_{\omega}\right) \boldsymbol{q}, \\
\dot{\boldsymbol{p}}_{i} & =\boldsymbol{C}^{T} \boldsymbol{w}_{p, i} \quad \forall i \in\{1, \ldots, N\} \\
\dot{\boldsymbol{b}}_{f} & =\boldsymbol{w}_{b f}, \\
\dot{\boldsymbol{b}}_{\omega} & =\boldsymbol{w}_{b \omega},
\end{aligned}
$$

where $\Omega(\cdot)$ maps an arbitrary rotational rate $\omega$ to the $4 \times 4$ matrix used for representing the corresponding quaternion rate:

$$
\boldsymbol{\Omega}: \boldsymbol{\omega} \mapsto \boldsymbol{\Omega}(\boldsymbol{\omega})=\left[\begin{array}{cccc}
0 & \omega_{z} & -\omega_{y} & \omega_{x} \\
-\omega_{z} & 0 & \omega_{x} & \omega_{y} \\
\omega_{y} & -\omega_{x} & 0 & \omega_{z} \\
-\omega_{x} & -\omega_{y} & -\omega_{z} & 0
\end{array}\right]
$$

While in principle the foot contacts are assumed to remain stationary, the white noise terms $\boldsymbol{w}_{p, i}$ in 17 with covariance parameter $\boldsymbol{Q}_{p, i}$ are added to the absolute foot positions in order to handle a certain amount of foot slippage. It is described in the body frame which allows tuning the magnitude of the noise terms in the different directions relative to the quadruped orientation (21). Furthermore, the noise parameter of a certain foothold is set to infinity (or to a very large value) whenever it has no ground contact. This enables the corresponding foothold to relocate and reset its position estimate when it regains ground contact, whereby the old foothold position is dropped from the estimation process. This is all that is required in order to handle intermittent contacts when stepping.

$$
\boldsymbol{Q}_{p, i}=\left[\begin{array}{ccc}
w_{p, i, x} & 0 & 0 \\
0 & w_{p, i, y} & 0 \\
0 & 0 & w_{p, i, z}
\end{array}\right]
$$

\section{Measurement Model}

Based on the kinematic model (2) a transformed measurement quantity is introduced for each leg $i$ :

$$
\begin{aligned}
\tilde{\boldsymbol{s}}_{i} & :=\operatorname{lkin}_{i}(\tilde{\boldsymbol{\alpha}}) \\
& \approx \operatorname{lkin}_{i}(\boldsymbol{\alpha})+\boldsymbol{J}_{\operatorname{lkin}, i} \boldsymbol{n}_{\alpha} \\
& \approx \boldsymbol{s}_{i} \underbrace{-\boldsymbol{n}_{s, i}+\boldsymbol{J}_{\mathrm{lkin}, i} \boldsymbol{n}_{\alpha}}_{\boldsymbol{n}_{i}} .
\end{aligned}
$$

The linearized noise effect from the encoders (1) and the noise from the foothold position are joined into a new measurement noise quantity $\boldsymbol{n}_{i}$ with covariance matrix $\boldsymbol{R}_{i}$ :

$$
\boldsymbol{R}_{i}=\boldsymbol{R}_{s}+\boldsymbol{J}_{\mathrm{lkin}, i} \boldsymbol{R}_{\alpha} \boldsymbol{J}_{\mathrm{lkin}, i}^{T}
$$

where $\boldsymbol{J}_{\mathrm{lkin}, i}$ is the Jacobian of the kinematics of leg $i$ with respect to the joint angles $\boldsymbol{\alpha}_{i}$ of the same leg:

$$
\boldsymbol{J}_{\text {lkin }, i}:=\frac{\partial \operatorname{lkin}_{i}(\boldsymbol{\alpha})}{\partial \boldsymbol{\alpha}_{i}} \quad i \in\{1, \ldots, N\} .
$$

$\tilde{\boldsymbol{s}}_{i}$ is the measurement of the position of the foot contact $i$ with respect to the body coordinate frame $\boldsymbol{B}$ which can also be expressed as the absolute position of the foot contact minus the absolute position of the robot rotated into the body frame.

$$
\tilde{\boldsymbol{s}}_{i}=\boldsymbol{C}\left(\boldsymbol{p}_{i}-\boldsymbol{r}\right)+\boldsymbol{n}_{i} .
$$

\section{Extended Kalman Filter Equations}

For the subsequent linearization and discretization of the above models, the following auxiliary quantity is introduced:

$$
\boldsymbol{\Gamma}_{n}:=\sum_{i=0}^{\infty} \frac{\Delta t^{i+n}}{(i+n) !} \boldsymbol{\omega}^{\times i}
$$

where the $(\cdot)^{\times}$superscript is used to represent the skewsymmetric matrix obtained from a vector. It draws on the series expansion of the matrix exponential. For $n=0$ it yields:

$$
\boldsymbol{\Gamma}_{0}=\sum_{i=0}^{\infty} \frac{\left(\Delta t \boldsymbol{\omega}^{\times}\right)^{i}}{i !}=\exp \left(\Delta t \boldsymbol{\omega}^{\times}\right) .
$$

This means that $\boldsymbol{\Gamma}_{0}$ represents the incremental rotation matrix if rotating an arbitrary coordinate frame with a rotational rate of $-\boldsymbol{\omega}$ for $\Delta t$ seconds. There exists a closed form expression for $\boldsymbol{\Gamma}_{n}$ that can be efficiently numerically evaluated (similar to Rodrigues' rotation formula). 
1) Prediction Step: A standard filtering convention is employed: at time step $k$ the a priori state estimate is represented by $\hat{\boldsymbol{x}}_{k}^{-}$, the a posteriori state estimate by $\hat{\boldsymbol{x}}_{k}^{+}$. Assuming zero-order hold for the measured quantities $\tilde{\boldsymbol{f}}_{k}$ and $\tilde{\boldsymbol{\omega}}_{k}$, and neglecting the effect of the incremental rotation, equations (14)-(19) can be discretized to:

$$
\begin{aligned}
\hat{\boldsymbol{r}}_{k+1}^{-} & =\hat{\boldsymbol{r}}_{k}^{+}+\Delta t \hat{\boldsymbol{v}}_{k}^{+}+\frac{\Delta t^{2}}{2}\left(\hat{\boldsymbol{C}}_{k}^{+T} \hat{\boldsymbol{f}}_{k}+\boldsymbol{g}\right), \\
\hat{\boldsymbol{v}}_{k+1}^{-} & =\hat{\boldsymbol{v}}_{k}^{+}+\Delta t\left(\hat{\boldsymbol{C}}_{k}^{+T} \hat{\boldsymbol{f}}_{k}+\boldsymbol{g}\right), \\
\hat{\boldsymbol{q}}_{k+1}^{-} & =\boldsymbol{\zeta}\left(\Delta t \hat{\boldsymbol{\omega}}_{k}\right) \otimes \hat{\boldsymbol{q}}_{k}^{+}, \\
\hat{\boldsymbol{p}}_{i, k+1}^{-} & =\hat{\boldsymbol{p}}_{i, k}^{+} \quad \forall i \in\{1, \ldots, N\}, \\
\hat{\boldsymbol{b}}_{f, k+1}^{-} & =\hat{\boldsymbol{b}}_{f, k}^{+}, \\
\hat{\boldsymbol{b}}_{\omega, k+1}^{-} & =\hat{\boldsymbol{b}}_{\omega, k}^{+},
\end{aligned}
$$

with the bias corrected IMU measurements

$$
\begin{aligned}
& \hat{\boldsymbol{f}}_{k}=\tilde{\boldsymbol{f}}_{k}-\hat{\boldsymbol{b}}_{f, k}^{+}, \\
& \hat{\boldsymbol{\omega}}_{k}=\tilde{\boldsymbol{\omega}}_{k}-\hat{\boldsymbol{b}}_{\omega, k}^{+} .
\end{aligned}
$$

In order to correctly propagate the covariance matrix through the state dynamics, a set of linear differential equations describing the error dynamics is derived from (14)-(19) where all higher order terms were neglected:

$$
\begin{aligned}
\dot{\boldsymbol{\delta}} \boldsymbol{r} & =\boldsymbol{\delta} \boldsymbol{v}, \\
\dot{\boldsymbol{\delta} \boldsymbol{v}} & =-\boldsymbol{C}^{T} \boldsymbol{f}^{\times} \boldsymbol{\delta} \boldsymbol{\phi}-\boldsymbol{C}^{T} \boldsymbol{\delta} \boldsymbol{b}_{f}-\boldsymbol{C}^{T} \boldsymbol{w}_{f}, \\
\dot{\boldsymbol{\delta}} \boldsymbol{\phi} & =-\boldsymbol{\omega}^{\times} \boldsymbol{\delta} \boldsymbol{\phi}-\boldsymbol{\delta} \boldsymbol{b}_{\omega}-\boldsymbol{w}_{\omega}, \\
\dot{\boldsymbol{\delta} \boldsymbol{p}_{i}} & =\boldsymbol{C}^{T} \boldsymbol{w}_{p, i} \quad \forall i \in\{1, \ldots, N\}, \\
\dot{\boldsymbol{\delta}} \boldsymbol{b}_{f} & =\boldsymbol{w}_{b f}, \\
\dot{\boldsymbol{\delta}} \boldsymbol{b}_{\omega} & =\boldsymbol{w}_{b \omega} .
\end{aligned}
$$

For the subsequent discretization, Van Loan's results [18] and the relation $\sqrt{28}$ can be applied to get the discrete linearized error dynamics matrix $\boldsymbol{F}_{k}$ and the discrete process noise covariance matrix $\boldsymbol{Q}_{k}$ (for readability only one foothold estimate is depicted):

$$
\boldsymbol{F}_{k}=\left[\begin{array}{cccccc}
\mathbb{I} & \Delta t \mathbb{I} & -\frac{\Delta t^{2}}{2} \hat{\boldsymbol{C}}_{k}^{+T} \hat{\boldsymbol{f}}_{k}^{\times} & 0 & -\frac{\Delta t^{2}}{2} \hat{\boldsymbol{C}}_{k}^{+T} & 0 \\
0 & \mathbb{I} & -\Delta t \hat{\boldsymbol{C}}_{k}^{+T} \hat{\boldsymbol{f}}_{k}^{\times} & 0 & -\Delta t \hat{\boldsymbol{C}}_{k}^{+T} & 0 \\
0 & 0 & \hat{\boldsymbol{\Gamma}}_{0, k}^{T} & 0 & 0 & -\hat{\boldsymbol{\Gamma}}_{1, k}^{T} \\
0 & 0 & 0 & \mathbb{I} & 0 & 0 \\
0 & 0 & 0 & 0 & \mathbb{I} & 0 \\
0 & 0 & 0 & 0 & 0 & \mathbb{I}
\end{array}\right],
$$

$$
\begin{array}{ccc}
{\left[\begin{array}{ccc}
\frac{\Delta t^{3}}{3} \boldsymbol{Q}_{f}+\frac{\Delta t^{5}}{20} \boldsymbol{Q}_{b f} & \frac{\Delta t^{2}}{2} \boldsymbol{Q}_{f}+\frac{\Delta t^{4}}{3} \boldsymbol{Q}_{b f} & 0 \\
\frac{\Delta t^{2}}{2} \boldsymbol{Q}_{f}+\frac{\Delta t^{4}}{8} \boldsymbol{Q}_{b f} & \Delta t \boldsymbol{Q}_{f}+\frac{\Delta t^{3}}{3} \boldsymbol{Q}_{b f} & 0 \\
0 & 0 & \Delta t \boldsymbol{Q}_{\omega}+\left(\hat{\boldsymbol{\Gamma}}_{3, k}+\hat{\boldsymbol{\Gamma}}_{3, k}^{T}\right) \boldsymbol{Q}_{b \omega} \\
0 & 0 & 0 \\
-\frac{\Delta t^{3}}{6} \boldsymbol{Q}_{b f} \hat{\boldsymbol{C}}_{k}^{+} & -\frac{\Delta t^{2}}{2} \boldsymbol{Q}_{b f} \hat{\boldsymbol{C}}_{k}^{+} & 0 \\
0 & 0 & -\boldsymbol{Q}_{b \omega} \hat{\boldsymbol{\Gamma}}_{2, k} \\
0 & -\frac{\Delta t^{3}}{6} \hat{\boldsymbol{C}}_{k}^{+T} \boldsymbol{Q}_{b f} & 0 \\
0 & -\frac{\Delta t^{2}}{2} \hat{\boldsymbol{C}}_{k}^{+T} \boldsymbol{Q}_{b f} & 0 \\
0 & 0 & -\hat{\boldsymbol{\Gamma}}_{2, k}^{T} \boldsymbol{Q}_{b \omega} \\
\Delta t \hat{\boldsymbol{C}}_{k}^{+T} \boldsymbol{Q}_{p} \hat{\boldsymbol{C}}_{k}^{+} & 0 & 0 \\
0 & \Delta t \boldsymbol{Q}_{b f} & 0 \\
0 & 0 & \Delta t \boldsymbol{Q}_{b \omega}
\end{array}\right]=\boldsymbol{Q}_{k} .} \\
\end{array}
$$

By linearly combining two Gaussian distributions the Extended Kalman Filter stipulates the following a priori estimate of the covariance matrix at the timestep $k+1$ :

$$
\boldsymbol{P}_{k+1}^{-}=\boldsymbol{F}_{k} \boldsymbol{P}_{k}^{+} \boldsymbol{F}_{k}^{T}+\boldsymbol{Q}_{k} .
$$

2) Update Step: The measurement residual, also called innovation, is the difference between actual measurements and their predicted value:

$$
\boldsymbol{y}_{k}:=\left(\begin{array}{c}
\tilde{\boldsymbol{s}}_{1, k}-\hat{\boldsymbol{C}}_{k}^{-}\left(\hat{\boldsymbol{p}}_{1, k}^{-}-\hat{\boldsymbol{r}}_{k}^{-}\right) \\
\vdots \\
\tilde{\boldsymbol{s}}_{N, k}-\hat{\boldsymbol{C}}_{k}^{-}\left(\hat{\boldsymbol{p}}_{N, k}^{-}-\hat{\boldsymbol{r}}_{k}^{-}\right)
\end{array}\right) .
$$

Considering the error states and again neglecting all higher order terms, it can be derived that the errors of the predicted leg kinematics measurements are given by:

$$
\begin{aligned}
\boldsymbol{s}_{i, k}-\hat{\boldsymbol{C}}_{k}^{-}\left(\hat{\boldsymbol{p}}_{i, k}^{-}-\hat{\boldsymbol{r}}_{k}^{-}\right) \approx & -\hat{\boldsymbol{C}}_{k}^{-} \boldsymbol{\delta} \boldsymbol{r}_{k}^{-}+\hat{\boldsymbol{C}}_{k}^{-} \boldsymbol{\delta} \boldsymbol{p}_{i, k}^{-} \\
& +\left(\hat{\boldsymbol{C}}_{k}^{-}\left(\boldsymbol{p}_{i, k}^{-}-\boldsymbol{r}_{k}^{-}\right)\right)^{\times} \boldsymbol{\delta} \boldsymbol{\phi}_{k}^{-} .
\end{aligned}
$$

With this the measurement Jacobian $\boldsymbol{H}_{k}$ can be evaluated:

$$
\begin{aligned}
\boldsymbol{H}_{k} & =\frac{\partial \boldsymbol{y}_{k}}{\partial \hat{\boldsymbol{x}}_{k}} \\
& =\left[\begin{array}{cccccccc}
-\hat{\boldsymbol{C}}_{k}^{-} & 0 & \left(\hat{\boldsymbol{C}}_{k}^{-}\left(\hat{\boldsymbol{p}}_{1, k}^{-}-\hat{\boldsymbol{r}}_{k}^{-}\right)\right)^{\times} & \hat{\boldsymbol{C}}_{k}^{-} & \cdots & 0 & 0 & 0 \\
\vdots & \vdots & \vdots & \vdots & \ddots & \vdots & \vdots & \vdots \\
-\hat{\boldsymbol{C}}_{k}^{-} & 0 & \left(\hat{\boldsymbol{C}}_{k}^{-}\left(\hat{\boldsymbol{p}}_{N, k}^{-}-\hat{\boldsymbol{r}}_{k}^{-}\right)\right)^{\times} & 0 & \cdots & \hat{\boldsymbol{C}}_{k}^{-} & 0 & 0
\end{array}\right] .
\end{aligned}
$$

Stacking the single measurement noise matrices (25) returns the total measurement noise matrix:

$$
\boldsymbol{R}_{k}=\left[\begin{array}{lll}
\boldsymbol{R}_{1, k} & & \\
& \ddots & \\
& & \boldsymbol{R}_{N, k}
\end{array}\right] .
$$

Finally the a priori state estimate can be merged with the current measurements, where the Extended Kalman Filter states the following update equations:

$$
\begin{aligned}
\boldsymbol{S}_{k} & :=\boldsymbol{H}_{k} \boldsymbol{P}_{k}^{-} \boldsymbol{H}_{k}^{T}+\boldsymbol{R}_{k}, \\
\boldsymbol{K}_{k} & :=\boldsymbol{P}_{k}^{-} \boldsymbol{H}_{k}^{T} \boldsymbol{S}_{k}^{-1}, \\
\boldsymbol{\Delta} \boldsymbol{x}_{k} & :=\boldsymbol{K}_{k} \boldsymbol{y}_{k}, \\
\boldsymbol{P}_{k}^{+} & :=\left(\boldsymbol{I}-\boldsymbol{K}_{k} \boldsymbol{H}_{k}\right) \boldsymbol{P}_{k}^{-}
\end{aligned}
$$

where $\boldsymbol{S}_{k}$ represents the innovation covariance, $\boldsymbol{K}_{k}$ the Kalman gain, $\boldsymbol{\Delta} \boldsymbol{x}_{k}$ the resulting correction vector and $\boldsymbol{P}_{k}^{+}$ the a posteriori estimate of the state covariance matrix. Given $\boldsymbol{\Delta} \boldsymbol{x}_{k}$ the state estimate can be updated. Again the orientation state requires special attention. Although the quaternion is of dimension 4, the extracted rotational correction $\Delta \phi_{k}$ has only 3 dimensions. It basically represents the $3 \mathrm{D}$ rotation vector that needs to be applied to correct the predicted quaternion:

$$
\hat{\boldsymbol{q}}_{k}^{+}=\boldsymbol{\zeta}\left(\boldsymbol{\Delta} \phi_{k}\right) \otimes \hat{\boldsymbol{q}}_{k}^{-} .
$$




\section{OBSERVABILITY ANALYSIS}

\section{A. Nonlinear Observability Analysis}

Analyzing the observability characteristics of the underlying nonlinear system reveals the theoretical limitations of state estimation and can validate the employed approach. Based on the paper of Hermann and Krener [8] a nonlinear observability analysis is performed. In order to remain analytically tractable robocentric coordinates are introduced. The coordinate transformation is bijective and will thus not change the observability characteristics. Given the current operating point by

$$
\boldsymbol{x}^{*}:=\left(\begin{array}{llllllll}
\boldsymbol{r}^{*} & \boldsymbol{v}^{*} & \boldsymbol{q}^{*} & \boldsymbol{p}_{1}^{*} & \cdots & \boldsymbol{p}_{N}^{*} & \boldsymbol{b}_{f}^{*} & \boldsymbol{b}_{\omega}^{*}
\end{array}\right)
$$

the following coordinate transformation is introduced:

$$
\boldsymbol{z}:=\left[\begin{array}{c}
\boldsymbol{s}_{1} \\
\vdots \\
\boldsymbol{s}_{N} \\
\overline{\boldsymbol{v}} \\
\overline{\boldsymbol{b}}_{\omega} \\
\overline{\boldsymbol{q}} \\
\overline{\boldsymbol{b}}_{f} \\
\overline{\boldsymbol{r}}
\end{array}\right]=\left[\begin{array}{c}
\boldsymbol{C}\left(\boldsymbol{p}_{1}-\boldsymbol{r}\right) \\
\vdots \\
\boldsymbol{C}\left(\boldsymbol{p}_{N}-\boldsymbol{r}\right) \\
\boldsymbol{C} \boldsymbol{v} \\
\boldsymbol{b}_{\omega}-\boldsymbol{b}_{\omega}^{*} \\
\boldsymbol{q} \otimes \boldsymbol{q}^{*-1} \\
\boldsymbol{b}_{f}-\boldsymbol{b}_{f}^{*} \\
\boldsymbol{C} \boldsymbol{r}
\end{array}\right]
$$

The quantities in (55) are ordered such that a nice row echelon form results. The corresponding prediction model (14)-(19) and measurement equation 27) will be transformed to

$$
\dot{\boldsymbol{z}}:=\left[\begin{array}{c}
\left(\boldsymbol{\omega}-\overline{\boldsymbol{b}}_{\omega}\right)^{\times} \boldsymbol{s}_{1}-\overline{\boldsymbol{v}} \\
\vdots \\
\left(\boldsymbol{\omega}-\overline{\boldsymbol{b}}_{\omega}\right)^{\times} \boldsymbol{s}_{N}-\overline{\boldsymbol{v}} \\
\left(\boldsymbol{\omega}-\overline{\boldsymbol{b}}_{\omega}\right)^{\times} \overline{\boldsymbol{v}}+\boldsymbol{f}-\overline{\boldsymbol{b}}_{f}+\overline{\boldsymbol{C}} \boldsymbol{C}^{*} \boldsymbol{g} \\
0 \\
\boldsymbol{\Omega}\left(\boldsymbol{\omega}-\overline{\boldsymbol{b}}_{\omega}\right) \overline{\boldsymbol{q}} \\
0 \\
\left(\boldsymbol{\omega}-\overline{\boldsymbol{b}}_{\omega}\right)^{\times} \overline{\boldsymbol{r}}+\overline{\boldsymbol{v}} \\
\tilde{\boldsymbol{s}}_{i}=\boldsymbol{s}_{i} \quad i \in\{1, \ldots, N\}
\end{array}\right],
$$

where all noise terms were disregarded as they have no influence on the observability and where $\bar{C}$ and $C^{*}$ represent the rotation matrices corresponding to $\overline{\boldsymbol{q}}$ and to $\boldsymbol{q}^{*}$, respectively.

The observability of the transformed system can now be analyzed. In contrast to the linear case, Lie-derivatives need to be computed in order to evaluate the observability matrix. By applying a few row-operations and by directly including the transformed operating point

$$
\boldsymbol{z}^{*}:=\left(\begin{array}{llllllllllll}
\boldsymbol{s}_{1}^{*} & \cdots & \boldsymbol{s}_{N}^{*} & \boldsymbol{C}^{*} \boldsymbol{v}^{*} & 0 & \left(\begin{array}{lllll}
0 & 0 & 0 & 1
\end{array}\right) & 0 & \boldsymbol{C}^{*} \boldsymbol{r}^{*}
\end{array}\right)
$$

the observability matrix can be converted into a row echelon form. For the sake of readability the * are dropped again:

$$
\begin{aligned}
& \mathcal{O}= {\left[\begin{array}{cccccccc}
\mathbb{1} & \cdots & 0 & 0 & 0 & 0 & 0 & 0 \\
\vdots & \ddots & \vdots & \vdots & \vdots & \vdots & \vdots & \vdots \\
0 & \cdots & \mathbb{I} & 0 & 0 & 0 & 0 & 0 \\
0 & \cdots & 0 & -\mathbb{I} & 0 & 0 & \boldsymbol{s}_{1}^{\times} & 0 \\
0 & \cdots & 0 & 0 & \mathbb{I} & -2(\boldsymbol{C g})^{\times} & \mathcal{O}_{1} & 0 \\
0 & \cdots & 0 & 0 & 0 & 2 \boldsymbol{\omega}^{\times}(\boldsymbol{C g})^{\times} & \mathcal{O}_{2} & 0 \\
0 & \cdots & 0 & 0 & 0 & 0 & \boldsymbol{\Delta} \boldsymbol{s}_{i, j}^{\times} & 0 \\
0 & \cdots & 0 & 0 & 0 & 0 & \boldsymbol{\Delta} \boldsymbol{s}_{i, j}^{\times} \boldsymbol{\omega}^{\times} & 0 \\
0 & \cdots & 0 & 0 & 0 & 0 & \boldsymbol{\Delta} \boldsymbol{s}_{i, j}^{\times} \boldsymbol{\omega}^{\times} & 0 \\
0 & \cdots & 0 & 0 & 0 & 0 & \mathcal{O}_{3} & 0 \\
0 & \cdots & 0 & 0 & 0 & 0 & \vdots & 0
\end{array}\right], \quad(59) } \\
& \mathcal{O}_{1}=-\boldsymbol{s}_{1}^{\times} \boldsymbol{\omega}^{\times}-2(\boldsymbol{C} \boldsymbol{v})^{\times}, \\
& \mathcal{O}_{2}=\left(\boldsymbol{s}_{1}^{\times} \boldsymbol{\omega}^{\times}+3(\boldsymbol{C v})^{\times}\right) \boldsymbol{\omega}^{\times}-\boldsymbol{\omega}^{\times}\left(\boldsymbol{s}_{1}^{\times} \boldsymbol{\omega}^{\times}+2(\boldsymbol{C v})^{\times}\right) \\
&-(\boldsymbol{C g})^{\times}-2 \boldsymbol{f}^{\times}, \\
& \mathcal{O}_{3}= \boldsymbol{\omega}^{\times}\left(\boldsymbol{s}_{1}^{\times} \boldsymbol{\omega}^{\times} \boldsymbol{\omega}^{\times}+5(\boldsymbol{C v})^{\times} \boldsymbol{\omega}^{\times}-4 \boldsymbol{f}^{\times}-3(\boldsymbol{C g})^{\times}\right) \\
&-\left(\boldsymbol{s}_{1}^{\times} \boldsymbol{\omega}^{\times} \boldsymbol{\omega}^{\times}+4(\boldsymbol{C} \boldsymbol{v})^{\times} \boldsymbol{\omega}^{\times}-3 \boldsymbol{f}^{\times}-2(\boldsymbol{C g})^{\times}\right) \boldsymbol{\omega}^{\times} \\
&-4 \boldsymbol{\omega}^{\times}(\boldsymbol{C} \boldsymbol{v}) \boldsymbol{\omega}^{T}, \\
& \boldsymbol{\mathcal { \Delta }} \boldsymbol{s}_{i, j}:=\boldsymbol{s}_{i}-\boldsymbol{s}_{j} .
\end{aligned}
$$

A full interpretation of this matrix is not within the scope of this paper. However, two essential points are emphasized. The four dimensional manifold composed of robot position and yaw angle (rotation around gravity vector $\boldsymbol{g}$ ) is always unobservable. This can be verified by looking at the tangential space spanned by the matrix

$$
\begin{aligned}
\overline{\boldsymbol{U}} & =\left[\begin{array}{lllllccc}
0 & \cdots & 0 & 0 & 0 & 0 & 0 & \mathbb{I} \\
0 & \cdots & 0 & 0 & 0 & \frac{1}{2}(\boldsymbol{C g})^{T} & 0 & 0
\end{array}\right]^{T}, \\
0 & =\mathcal{O} \overline{\boldsymbol{U}}
\end{aligned}
$$

Infinitesimal errors $\Delta z=\bar{U} \epsilon$ lying within the subspace of $\overline{\boldsymbol{U}}$ cannot be detected. Transforming this back to our original coordinates yields the tangential space

$$
\boldsymbol{U}=\left[\begin{array}{cccccccc}
\boldsymbol{C}^{T} & 0 & 0 & \boldsymbol{C}^{T} & \cdots & \boldsymbol{C}^{T} & 0 & 0 \\
\boldsymbol{g}^{T} \boldsymbol{r}^{\times} & \boldsymbol{g}^{T} \boldsymbol{v}^{\times} & \boldsymbol{g}^{T} \boldsymbol{C}^{T} & \boldsymbol{g}^{T} \boldsymbol{p}_{1}^{\times} & \cdots & \boldsymbol{g}^{T} \boldsymbol{p}_{N}^{\times} & 0 & 0
\end{array}\right]^{T}
$$

where the upper row corresponds to a 3 dimensional translation of the inertial coordinate frame and where the lower row corresponds to a rotation of the inertial coordinate frame around the gravity axis $\boldsymbol{g}$.

The second point is that in some cases, the rank loss associated with the unobservable manifold can increase by up to 5 additional ranks. Table I depicts some of the cases. All cases which induce a rank loss require some singularities. It can thus be stated that statistically almost surely the unobservable space will be limited to absolute position and yaw angle (except for the case where there is no ground contact at all). Note that if the bias estimation is excluded, the unobservable subspace will be invariantly of rank four.

Unfortunately, typical operating points can lie very close to singular cases. The upper highlighted row in table I represents 


\begin{tabular}{|c|c|c|c|r|}
$\boldsymbol{\omega}$ & $\boldsymbol{f}$ & $\boldsymbol{v}$ & $\boldsymbol{s}_{1}, \ldots, \boldsymbol{s}_{N}$ & Rank loss \\
\hline $\boldsymbol{\omega} \cdot \boldsymbol{C g} \neq 0$ & $*$ & $*$ & not co-linear & 0 \\
\hline $\boldsymbol{\omega} \cdot \boldsymbol{C g} \neq 0$ & det $\boldsymbol{O}_{3} \neq 0$ & at least one contact & 0 \\
\hline $\boldsymbol{\omega} \cdot \boldsymbol{C g}=0$ & $*$ & $*$ & at least one contact & $\geq 1$ \\
\hline 0 & $*$ & $*$ & at least one contact & $\geq 2$ \\
\hline 0 & $*$ & $*$ & not co-linear & 2 \\
\hline 0 & 0 & $*$ & $\boldsymbol{s}_{1}=\ldots=\boldsymbol{s}_{N}$ & 3 \\
\hline 0 & $-1 / 2 \boldsymbol{C g}$ & $*$ & $\boldsymbol{s}_{1}=\ldots=\boldsymbol{s}_{N}$ & 5 \\
\hline
\end{tabular}

TABLE I

ESTIMATION SCENARIOS AND CORRESPONDING RANK LOSS.

the case where the robot has at least 3 non co-linear ground contacts and where the rotation axis is not perpendicular to the gravity vector. The lower highlighted row represents the corresponding singular case where $\boldsymbol{\omega}=0$ inducing a rank loss of 2. This proximity to singular cases can cause bad convergence quality. For this reason the filter is implemented in such a manner that the estimation of the accelerometer and gyroscope biases can be enabled or disabled at runtime. Thereby it is possible to disable the bias estimation for critical tasks. On the other hand special maneuvers can be derived from the conditions in table $\Pi$ which can properly estimate the bias states.

\section{B. Observability Analysis of the Extended Kalman Filter}

The filter makes use of a linearized and discretized version of the nonlinear system model:

$$
\begin{aligned}
\boldsymbol{x}_{k+1} & =\boldsymbol{F}_{k} \boldsymbol{x}_{k}+\boldsymbol{w}_{l i n, k}, \\
\boldsymbol{y}_{k} & =\boldsymbol{H}_{k} \boldsymbol{x}_{k}+\boldsymbol{n}_{l i n, k},
\end{aligned}
$$

where errors caused by linearization or discretization are incorporated in the noise terms $\boldsymbol{w}_{l i n, k}$ and $\boldsymbol{n}_{l i n, k}$. The corresponding observability analysis will be performed by applying the concept of local observability matrices [1]: similar to the time-invariant case the observable subspace can be derived by analyzing the subspace spanned by the rows of a local observability matrix:

$$
\mathcal{M}=\left[\begin{array}{c}
\boldsymbol{H}_{k} \\
\boldsymbol{H}_{k+1} \boldsymbol{F}_{k} \\
\boldsymbol{H}_{k+2} \boldsymbol{F}_{k+1} \boldsymbol{F}_{k} \\
\boldsymbol{H}_{k+3} \boldsymbol{F}_{k+2} \boldsymbol{F}_{k+1} \boldsymbol{F}_{k} \\
\vdots
\end{array}\right] .
$$

The observability characteristics of the discrete linear timevarying system 67)-68 can differ from those of the underlying nonlinear system (14)-(19), 27). This discrepancy can be caused by linearization/discretization effects as well as by noise effects. The effect of noise becomes particularly evident when contemplating the observability characteristics of a corresponding noiseless (ideal) system. For the presented system the effect of noise renders the yaw angle observable by preventing the evaluation of the Jacobians $\boldsymbol{F}_{k}$ and $\boldsymbol{H}_{k}$ around the true state and thereby increasing the numerical rank of the local observability matrix $\mathcal{M}$. The spurious appearance of new observable states is strongly objectionable as it results in overconfident state estimation. The magnitude of this inconsistency depends on the noise ratio, but in the long run, it will always deteriorate the state estimate.
The above phenomenon has been observed earlier in the context of EKF-SLAM [9, 12]. Huang et al. [10] introduced the Observability Constrained Extended Kalman Filter in order to tackle this issue. The approach in this paper goes much along their idea: the unobservable subspace of the nonlinear system (66) should also be unobservable in the linearized and discretized system 67 -68. Mathematically, this can be imposed by adding the following constraint:

$$
\mathcal{M} U=0 .
$$

In order to meet this constraint Huang et al. evaluate the Jacobians at special operating points: instead of using the actual state estimate they use slightly altered values.

The approach in this paper tackles the observability problem by exploiting the following observation: the noiseless case does meet the constraint (70) because it perfectly fulfills the prediction equations 30 - 35 and thus the appropriate terms are canceled out. For the presented filter it suffices if the following constraints are introduced (where ${ }^{*}$ denotes the states or measurements around which Jacobians are evaluated):

$$
\begin{aligned}
\boldsymbol{r}_{k+1}^{*} & =\boldsymbol{r}_{k}^{*}+\Delta t \boldsymbol{v}_{k}^{*}+\frac{\Delta t^{2}}{2}\left(\boldsymbol{C}_{k}^{* T} \boldsymbol{f}_{k, 1}^{*}+\boldsymbol{g}\right), \\
\boldsymbol{v}_{k+1}^{*} & =\boldsymbol{v}_{k}^{*}+\Delta t\left(\boldsymbol{C}_{k}^{* T} \boldsymbol{f}_{k, 2}^{*}+\boldsymbol{g}\right), \\
\boldsymbol{q}_{k+1}^{*} & =\boldsymbol{\zeta}\left(\boldsymbol{\omega}_{k}^{*}\right) \otimes \boldsymbol{q}_{k}^{*} \\
\boldsymbol{p}_{i, k+1}^{*} & =\boldsymbol{p}_{i, k}^{*} \quad \forall i \in\{1, \ldots, N\} .
\end{aligned}
$$

Both, filter state and IMU measurements, are allowed to differ from their actual estimated quantities. However, in order to keep the linearization errors small the linearization point should remain as close as possible to the estimated state. Thus, given the timestep $l_{i}$ of the last touch-down event of foot $i$, the first-ever available estimate is chosen for the linearization:

$$
\begin{aligned}
& \boldsymbol{r}_{k}^{*}=\boldsymbol{r}_{k}^{-}, \quad \boldsymbol{v}_{k}^{*}=\boldsymbol{v}_{k}^{-}, \quad \boldsymbol{q}_{k}^{*}=\boldsymbol{q}_{k}^{-}, \\
& \boldsymbol{p}_{i, k}^{*}=\boldsymbol{p}_{i, l_{i}}^{-} \quad \forall i \in\{1, \ldots, N\} .
\end{aligned}
$$

This is in analogy to the First-Estimates Jacobian EKF of Huang et al. [9]. But, in general, the prediction constraints (71)-73) are still not met. For this reason the additional terms $\boldsymbol{f}_{k, 1}^{*}, \boldsymbol{f}_{k, 2}^{*}$ and $\boldsymbol{\omega}_{k}^{*}$ were introduced. Now, by choosing

$$
\begin{aligned}
\boldsymbol{f}_{k, 1}^{*} & =\boldsymbol{C}_{k}^{* T}\left(\frac{\boldsymbol{r}_{k+1}^{*}-\boldsymbol{r}_{k}^{*}-\Delta t \boldsymbol{v}_{k}^{*}}{0.5 \Delta t^{2}}-\boldsymbol{g}\right), \\
\boldsymbol{f}_{k, 2}^{*} & =\boldsymbol{C}_{k}^{* T}\left(\frac{\boldsymbol{v}_{k+1}^{*}-\boldsymbol{v}_{k}^{*}}{\Delta t}-\boldsymbol{g}\right), \\
\boldsymbol{\omega}_{k}^{*} & =\boldsymbol{\zeta}^{-1}\left(\boldsymbol{q}_{k+1}^{*} \otimes \boldsymbol{q}_{k}^{*-1}\right)
\end{aligned}
$$

all constraints can be easily met. The above quantities represent the IMU measurements that would arise when considering two subsequent filter prediction states at timestep $k$ and $k+1$. Because the acceleration related measurements can differ if evaluated based on the position prediction or on the velocity prediction, two terms were introduced. This permits to keep the computation of the linearization quantities simple and avoids complex optimization algorithms or oscillation provoking bindings between subsequent linearization points. 
Computing the Jacobians $\boldsymbol{F}_{k}$ and $\boldsymbol{H}_{k}$ using the supplementary linearization quantities and evaluating the corresponding local observability matrix 69 yields:

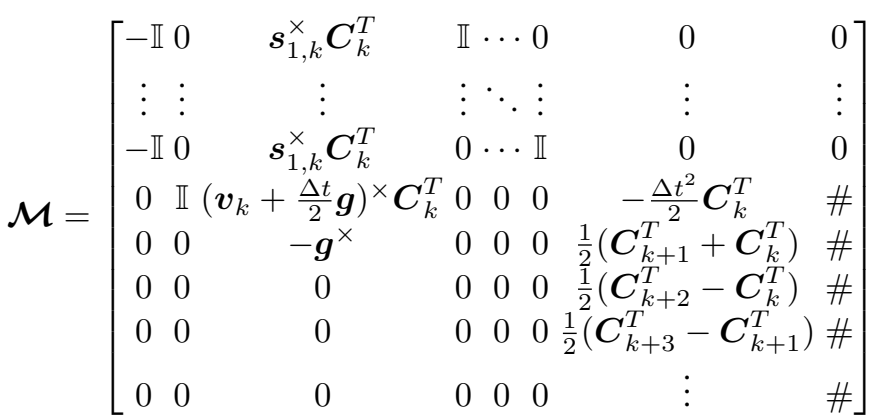

whereby it is simple to test that the observability constraint (70) is satisfied. As a last side note: similarly to the nonlinear case, observability rank loss will again be induced when $\boldsymbol{\omega} \equiv 0$ and thus

$$
C_{k+2}^{T}-C_{k}^{T}=0 .
$$

\section{RESUlts AND DisCUSSION}

Experiments are performed in simulation and on a real platform, whereby a series-elastic actuated quadruped is stabilized by a virtual model control approach [11] using the feedback of the pose estimator. The estimation of accelerometer and gyroscope biases is always enabled. In a first experiment the filter behavior is evaluated for a dynamic trotting gait within a simulation environment including realistic noise levels. Fig. 2 shows results from a $15 \mathrm{~s}$ trajectory with a reference forward speed of $0.4 \mathrm{~m} / \mathrm{s}$. The uncertainties of the robot and of the foothold positions are represented by the corresponding $1 \sigma$ ellipses. The effects of unobservable absolute position and yaw angle can clearly be perceived. The leg kinematics measurements directly correlate the estimate of the main body position and the estimates of the foothold positions and thereby strongly limit the drift. Moreover, considering the correlations induced by the prediction model, the filter is able to properly correct the estimated quantities rendering the inclination angles and the velocities fully observable. Based on the resulting state estimate the quadruped can stabilize itself in a highly dynamic gait.

The second set of results is collected on a real platform. During the experiment independent ground truth measurements are provided by an external visual tracking system. A $60 \mathrm{~s}$ long static walking sequence where the robot moves approximately one meter forward is evaluated. By pushing and tilting the robot external disturbances are imposed on the slow locomotion pattern. Because the position is not fully observable, a slight drift occurs for the corresponding estimates (see Fig. 3, it can amount up to roughly $10 \%$ of the traveled distance. Notable sources for the drift are the inaccurate leg kinematics and the fault-prone contact detection. The slightly longer actual robot shank explains the shorter estimated traveled distance (x direction). On the other hand, small perturbations are closely tracked by the filter. This is attested by very precise velocity estimates yielding RMS error values of less than $0.02 \mathrm{~m} / \mathrm{s}$ (see Fig. 4). Like the velocity states, the roll and pitch angles are fully observable as well and exhibit also very small estimation errors (see Fig. 5). The drift of the yaw angle is almost imperceivable. For all estimates the corresponding $3 \sigma$ covariance-hull is plotted. Except for the $\mathrm{x}$-position estimate, where model inaccuracies induce a significant offset, all estimate errors remain within the covariance-hull and thus validate the consistency of the presented approach.

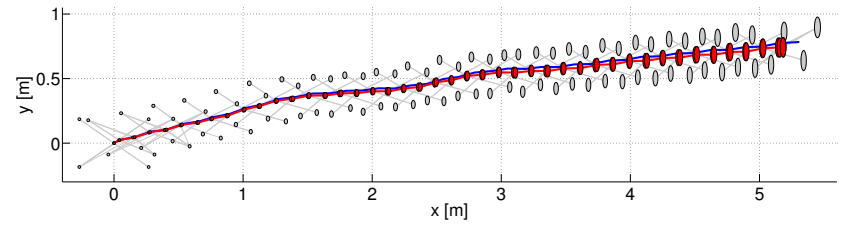

Fig. 2. 2D view of a $5 \mathrm{~m}$ trotting sequence in simulation. Blue: ground-truth body trajectory. Red ellipses: successive position estimates of the robot's main body. Grey ellipses: estimates of the foothold positions. In both cases the ellipses are scaled depending on the corresponding standard deviation $(1 \sigma)$. The position error at the end amounts to less than $5 \%$ of the traveled distance.
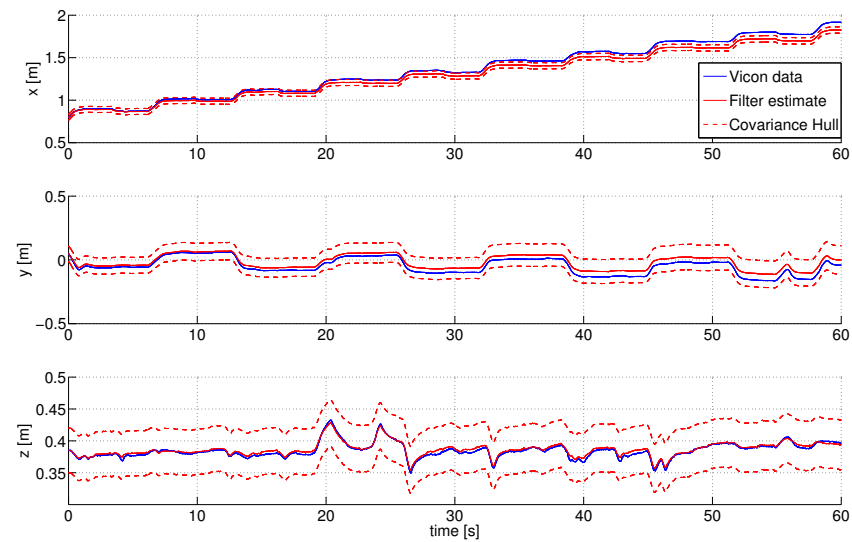

Fig. 3. Comparison between estimated position and the motion capture system's position outputs. All three positions are affected by some drift, amounting up to $10 \%$ of the traveled distance.
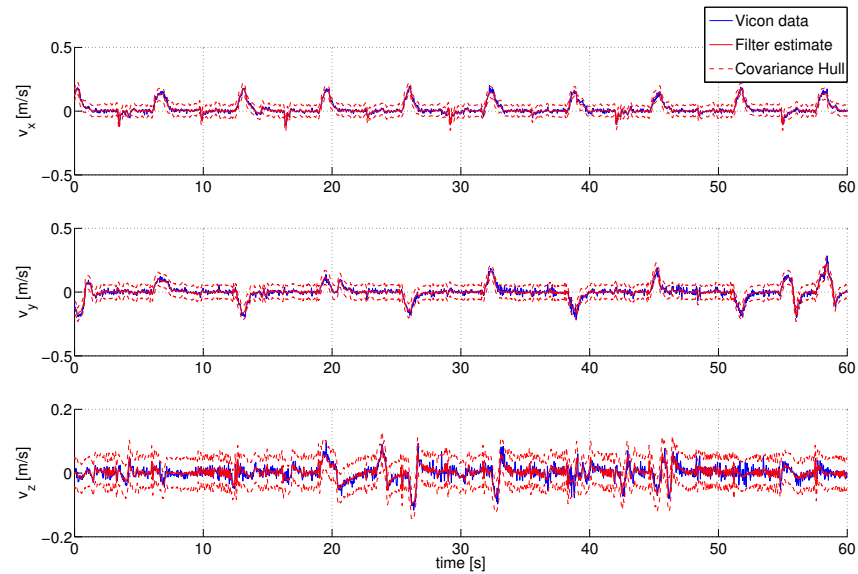

Fig. 4. Comparison between estimated velocity and the motion capture system's numerical position derivatives. All three velocity estimates are fully observable and consequently can be tracked very accurately. The resulting RMS error values are $0.0111 \mathrm{~m} / \mathrm{s}, 0.0153 \mathrm{~m} / \mathrm{s}$ and $0.0126 \mathrm{~m} / \mathrm{s}$. 

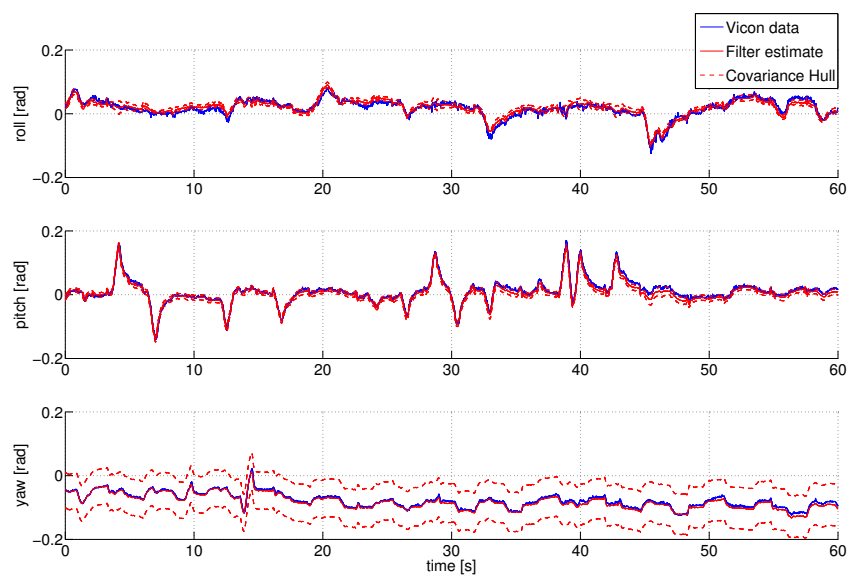

Fig. 5. Comparison between estimated roll, pitch and yaw angle and the motion capture system's orientation outputs. Roll and pitch angle are fully observable and the filter produces very precise corresponding estimates, with angular error RMS of less than $0.5 \mathrm{deg}(0.0088 \mathrm{rad}$ and $0.0073 \mathrm{rad})$. The yaw angle drift is almost unnoticeable.

\section{CONCLUSION AND Future WORK}

This paper presents a pose estimator for legged robots. It fuses information from leg kinematics and IMU data, whereby the model equations are kept simple and precise, and only a minimum of assumptions is introduced (mainly limited foot slippage). The filter can handle unknown terrain and arbitrary locomotion gaits. Through an observability analysis, it was shown that for non-degenerate cases only absolute position and yaw angle are not observable. Consequently, the roll and pitch angles as well as the robot's velocity can be accurately tracked, which was confirmed by the experimental results. Compared to proprioceptive sensor setups only, the obtained state estimate attains an unpreceded level of precision. The very generic formulation enables the filter to be extended with further sensory measurements and allows its implementation on various kinds of legged platforms.

Future work will include handling the unobservable states. Different approaches like introducing coordinate transformations, partitioning the unobservable manifold or implementing pseudo-measurements could be evaluated. Fusion with exteroceptive sensors will also be investigated. More aggressive locomotion needs to be further tested: while it has been validated in simulation, future work will include dynamic walking on the real quadruped platform.

\section{REFERENCES}

[1] Z. Chen, K. Jiang, and J.C. Hung. Local observability matrix and its application to observability analyses In Industrial Electronics Society, 16th Annual Conference of IEEE, Nov. 1990.

[2] A. Chilian, H. Hirschmüller, and M. Görner. Multisensor data fusion for robust pose estimation of a sixlegged walking robot. In Intelligent Robots and Systems, IEEE/RSJ International Conference on, Sep. 2011.

[3] S. Chitta, P. Vernaza, R. Geykhman, and D.D. Lee. Proprioceptive localization for a quadrupedal robot on known terrain In Robotics and Automation, IEEE International Conference on, Apr. 2007.

[4] J. A. Cobano, J. Estremera, and P. Gonzalez de Santos. Location of legged robots in outdoor environments Robotics and Autonomous Systems, 56:751-761, 2008.

[5] N. El-Sheimy, Haiying Hou, and Xiaoji Niu. Analysis and modeling of inertial sensors using allan variance Instrumentation and Measurement, IEEE Transactions on, 57(1):140-149, Jan. 2008.

[6] B. Gassmann, F. Zacharias, J.M. Zöllner, and R. Dillmann. Localization of walking robots. In Robotics and Automation, IEEE Int. Conf. on, Apr. 2005.

[7] O. Gur and U. Saranli. Model-based proprioceptive state estimation for spring-mass running. In Proceedings of Robotics: Science and Systems, Jun. 2011.

[8] R. Hermann and A. Krener. Nonlinear controllability and observability. Automatic Control, IEEE Transactions on, 22(5):728-740, Oct. 1977.

[9] G.P. Huang, A.I. Mourikis, and S.I. Roumeliotis. Analysis and improvement of the consistency of extended kalman filter based slam In Robotics and Automation, IEEE International Conference on, May 2008.

[10] Guoquan P. Huang, Anastasios I. Mourikis, and Stergios I. Roumeliotis. Observability-based rules for designing consistent ekf slam estimators International Journal of Robotics Research, 29:502-528, Apr. 2010.

[11] M. Hutter, C. Gehring, M. Bloesch, M.A. Hoepflinger, C.D. Remy, and R. Siegwart. StarlETH: A compliant quadrupedal robot for fast, efficient, and versatile locomotion. In Climbing and Walking Robots, International Conference on, Jul. 2012.

[12] S.J. Julier and J.K. Uhlmann. A counter example to the theory of simultaneous localization and map building. In Robotics and Automation, IEEE Int. Conf. on, May 2001.

[13] P.C. Lin, H. Komsuoglu, and D.E. Koditschek. A leg configuration measurement system for full-body pose estimates in a hexapod robot Robotics, IEEE Transactions on, 21(3):41-422, Jun. 2005.

[14] P.C. Lin, H. Komsuoglu, and D.E. Koditschek. Sensor data fusion for body state estimation in a hexapod robot with dynamical gaits Robotics, IEEE Transactions on, 22(5):932-943, Oct. 2006.

[15] M. Reinstein and M. Hoffmann. Dead reckoning in a dynamic quadruped robot: Inertial navigation system aided by a legged odometer In Robotics and Automation, IEEE International Conference on, May 2011.

[16] S.P.N. Singh, P.J. Csonka, and K.J. Waldron. Optical flow aided motion estimation for legged locomotion In Intelligent Robots and Systems, IEEE/RSJ International Conference on, Oct. 2006.

[17] F.-L. Tong and M.Q.-H. Meng. Localization for legged robot with single low-resolution camera using genetic algorithm. In Integration Technology, IEEE, Mar. 2007.

[18] C. Van Loan. Computing integrals involving the matrix exponential. Automatic Control, IEEE Transactions on, 23(3):395-404, Jun. 1978. 